For Publisher's use

\title{
ELECTROWEAK SYMMETRY BREAKING CIRCA 2005
}

\author{
S. DAWSON \\ Physics Department, Brookhaven National Laboratory, Upton, NY, 11973, USA \\ E-mail: dawson@bnl.gov
}

\begin{abstract}
Recent progress in both the experimental and theoretical explorations of electroweak symmetry breaking is surveyed.
\end{abstract}

\section{Introduction}

Particle physicists have a Standard Model of electroweak interactions which describes a large number of measurements extraordinarily well at energies on the few hundred $\mathrm{GeV}$ scale. In fact, we have become extremely blasé about tables such as that of Fig. 1, ${ }^{1}$ which shows an impressive agreement between experiment and theory. Virtual probes, using the sensitivity of rare decays to high scale physics, are also in good agreement with the predictions of the Standard Model. This agreement, however, assumes the existence of a light, scalar Higgs boson, without which the theory is incomplete. There has thus been an intense experimental effort at the Tevatron aimed at discovering either the Standard Model Higgs boson or one of the Higgs bosons associated with the minimal supersymmetric model (MSSM).

In the Standard Model, using $G_{F}, \alpha$, and $M_{Z}$ as inputs, along with the fermion masses, the $W$ mass is a predicted quantity. The comparison between the prediction and the measured value can not only be used to check the consistency of the theory, but also to infer limits on possible extentions of the Standard Model. The relationship between $M_{W}$ and $M_{t}$ is shown in Fig. 2. The curve labelled "old" does not include the new values (as of Summer, 2005), for the $W$ mass and width from LEP-2 and the new mass of the top quark from the Tevatron. (These new values are reflected in Fig. 1.)

The measurements of Fig. 1 can be used to extract limits on the mass of a Standard
Model Higgs boson. The limit on the Higgs boson mass depends quadratically on the top quark mass and logarithmically on the Higgs boson mass, making the limit exquisitely sensitive to the top quark mass. The limit is also quite sensitive to which pieces of data are included in the analysis. The fit of Fig. 2 includes only the high energy data and so does not include results from $\mathrm{NuTeV}$ or atomic parity violation.

The precision electroweak measurements of Fig. 1 give a 95\% confidence level upper limit on the value of the Higgs boson mass of, ${ }^{1}$

$$
M_{H}<186 \mathrm{GeV} \text {. }
$$

If the LEP-2 direct search limit of $M_{H}>$ $114 \mathrm{GeV}$ is included, the limit increases to

$$
M_{H}<219 \mathrm{GeV} \text {. }
$$

Both CDF and D0 have presented experimental limits on the production rate for a Standard Model Higgs boson, which are shown in Fig. $\quad 3 .^{2}$ For most channels, the limits are still several orders of magnitude away from the predicted cross sections in the Standard Model. With an integrated luminosity of $4 \mathrm{fb}^{-1}\left(8 \mathrm{fb}^{-1}\right)$, the $95 \%$ exclusion limit will increase to $M_{H}>130 \mathrm{GeV}$ $\left(M_{H}>135 \mathrm{GeV}\right)$. A much more optimistic viewpoint is to note that with $4 \mathrm{fb}^{-1}$ there is a $35 \%$ chance that the Tevatron will find $3 \sigma$ evidence for a Higgs boson with a mass up to $M_{H}=130 \mathrm{GeV}$.

Despite the impressive agreement between the precision electroweak data and the theoretical predictions of the Standard 


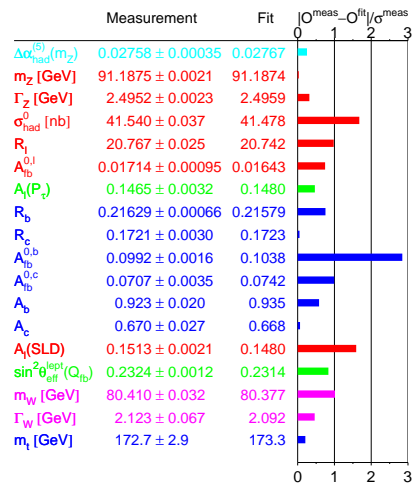

Figure 1. Precision electroweak measurements and the best theoretical fit to the Standard Model as of September, 2005. Also shown is the deviation of the fit for each measurement from the value predicted using the parameters of the central value of the fit[1]

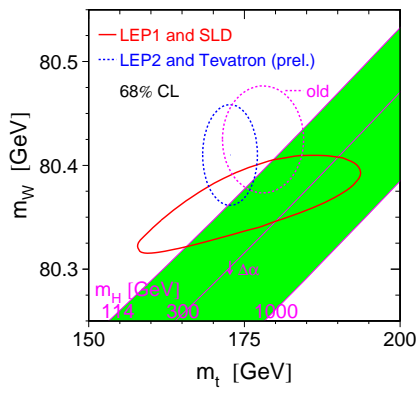

Figure 2. The relationship between $M_{W}$ and $M_{t}$ in the Standard Model. The curve labelled "old" does not include the Summer, 2005 updates on the $W$ boson mass and width from LEP-2 and the new top quark mass from the Tevatron[1].

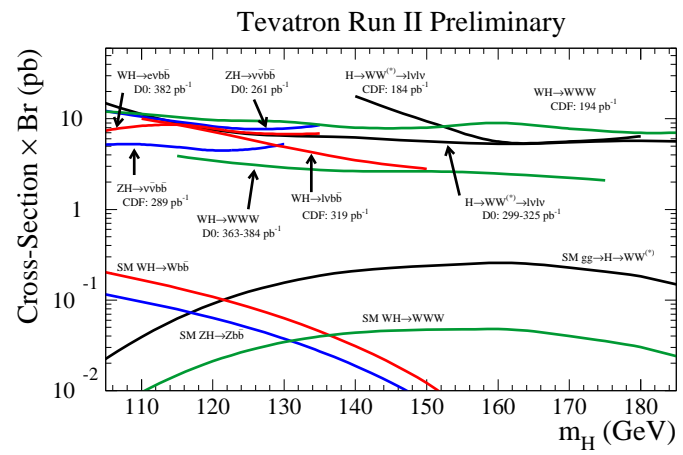

Figure 3. CDF and D0 limits on the production cross section times the branching ratios for various Higgs boson production channels as a function of the Higgs boson mass, along with the Standard Model expectations for each channel[2].

Model with a light Higgs boson, theorists have been busy inventing new models where mechanisms other than a light Higgs boson are responsible for the electroweak symmetry breaking. We begin in Section 2 by reviewing the theoretical arguments for the existence of a Higgs boson and continue in Section 3 to discuss the reasons why a light Higgs boson is unattractive to many theorists. In the following sections, we review a sampling of models of electroweak symmetry breaking.

\section{Who needs a Higgs Boson?}

The Standard Model requires a Higgs boson for consistency with precision electroweak data, as is clear from Fig. 2. The Standard Model Higgs boson also serves two additional critical functions.

The first is to generate gauge invariant masses for the fermions. Since left- $\left(\psi_{L}\right)$ and right- $\left(\psi_{R}\right)$ handed fermions transform differently under the chiral $S U(2)_{L} \times U(1)_{Y}$ gauge groups, a mass term of the form

$$
L_{\text {mass }} \sim m_{f}\left(\bar{\psi}_{L} \psi_{R}+\bar{\psi}_{R} \psi_{L}\right)
$$



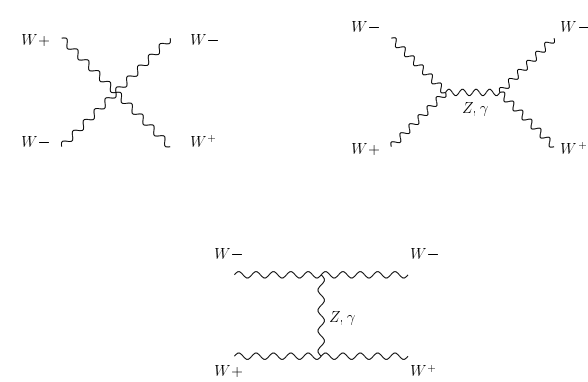

Figure 4. Feynman diagrams contributing to the process $W^{+} W^{-} \rightarrow W^{+} W^{-}$with the Higgs boson removed from the theory.

is forbidden by the gauge symmetry. A Higgs doublet, $\Phi$, with a vacuum expectation value, $v$, generates a mass term of the required form,

$$
L_{\text {mass }} \sim \frac{m_{f}}{v}\left(\bar{\psi}_{L} \Phi \psi_{R}+\bar{\psi}_{R} \Phi^{\dagger} \psi_{L}\right) .
$$

The second important role of the Standard Model Higgs boson is to unitarize the gauge boson scattering amplitudes. The $J=0$ partial wave amplitude for the process $W^{+} W^{-} \rightarrow W^{+} W^{-}$(Fig. 4) grows with energy when the Higgs boson is not included in the amplitude and violates partial wave unitarity at an energy around $E \sim 1.6 \mathrm{TeV} .^{3}$ The Higgs boson has just the right couplings to the gauge bosons to restore partial wave unitarity as long as the Higgs boson mass is less than around $M_{H}<800 \mathrm{GeV}$. With a Higgs boson satisfying this limit, the Standard Model preserves unitarity at high energies and is weakly interacting.

\section{Problems in Paradise}

The Standard Model is theoretically unsatisfactory, however, because when loop corrections are included, the Higgs boson mass contains a quadratic dependence on physics at some unknown higher energy scale, $\Lambda$. When the one-loop corrections to the Higgs boson
Table 1. Representative limits (90\% c.l.) on the scale of new dimension- 6 operators corresponding to $L=$ $\mathcal{O}_{i} / \Lambda^{2}[4]$.

\begin{tabular}{|c|c|c|}
\hline & Operator, $\mathcal{O}_{i}$ & $\Lambda_{\min }(\mathrm{TeV})$ \\
\hline LEP & $H^{\dagger} \tau H W_{\mu \nu}^{a} B^{\mu \nu}$ & 10 \\
\hline LEP-2 & $\bar{e} \gamma_{\mu} \bar{e} \gamma^{\mu} l$ & 5 \\
\hline Flavor & $H^{\dagger} \bar{d}_{R} \sigma_{\mu \nu} q_{L} F^{\mu \nu}$ & 9 \\
\hline
\end{tabular}

mass, $\delta M_{H}^{2}$, are computed we find,

$$
\begin{aligned}
\delta M_{H}^{2} & =\frac{G_{F} \Lambda^{2}}{4 \sqrt{2} \pi^{2}}\left(6 M_{W}^{2}+3 M_{Z}^{2}+M_{H}^{2}-12 M_{t}^{2}\right) \\
& \sim-\left(\frac{\Lambda}{.7 T e V} 200 \mathrm{GeV}\right)^{2} .
\end{aligned}
$$

In order to have a light Higgs boson as required by the precision electroweak measurements, the scale $\Lambda$ must be near $1 \mathrm{TeV}$. The quantum corrections thus suggest that there must be some new physics lurking at the $\mathrm{TeV}$ scale.

We therefore need new physics at the $1 \mathrm{TeV}$ scale to get a light Higgs boson. However, much of the possible new physics at this scale is already excluded experimentally. A model independent analysis which looked at various dimension- 6 operators found that typically new physics cannot occur below a scale $\Lambda>5 \mathrm{TeV}$. A representative sampling of limits on possible dimension- 6 operators is shown in Table 1 and a more complete list can be found in Ref.[3]. This tension between needing a low scale $\Lambda$ for new physics in order to get a light Higgs boson and the experimental exclusion of much possible new physics at the $\mathrm{TeV}$ scale has been dubbed the "little hierarchy problem". However, a global fit to 21 flavor- and CP- conserving operators found that there are certain directions in parameter space where the limit on $\Lambda$ can be lowered considerably ${ }^{5}$ (even to below $1 \mathrm{TeV}$ ) raising the possibility that in specific models the "little hierarchy problem" may not be a problem at all.

In recent years, there have been a vari- 
ety of creative new models constructed which attempt to find a mechanism to lower the scale $\Lambda$, while at the same time not violating the existing experimental limits. Supersymmetric models are the trusty standard for addressing this problem and we discuss progress and variations on the minimal supersymmetric model in the next section. In the following sections, we discuss attempts to address electroweak symmetry breaking with Little Higgs models ${ }^{7,8}$ and with Higgsless models. ${ }^{6}$ There are many other novel models for electroweak symmetry breakingfat Higgs models, ${ }^{9}$ strong electroweak symmetry breaking ${ }^{10}$ (and many more!) - which will not be addressed here due to space limitations.

\section{Supersymmetry}

The classic model of new physics at the $\mathrm{TeV}$ scale is supersymmetry, where a cancellation between the contributions of the Standard Model particles and the new partner particles of a supersymmetric model keeps the Higgs boson mass at the $\mathrm{TeV}$ scale. This cancellation occurs as long as the supersymmetric partner particles have masses on the order of the weak scale. For example, the top quark contribution to Eq. 5 becomes, ${ }^{13}$

$$
\delta M_{H}^{2} \sim G_{F} \Lambda^{2}\left(M_{t}^{2}-\tilde{m}_{t 1, t 2}^{2}\right),
$$

where $\tilde{m}_{t 1, t 2}$ are the masses of the scalar partners of the top quark.

The simplest version of a supersymmetric model, the MSSM, has many positive aspects:

- The MSSM predicts gauge coupling unification at the GUT scale.

- The MSSM contains a dark matter candidate, the LSP (Lightest Supersymmetric Particle).

- The MSSM predicts a light Higgs boson, $M_{H}<140 \mathrm{GeV}$.

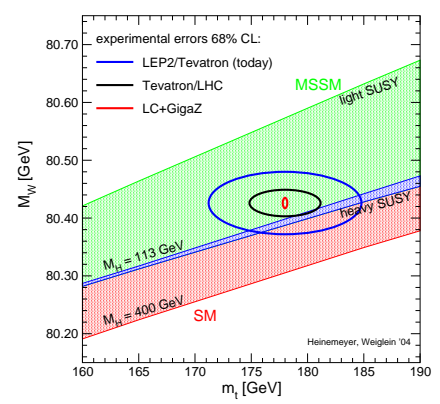

Figure 5. Fit to precision electroweak data in the MSSM. The curve labelled heavy SUSY assumes the supersymmetric parameters are set at $2 \mathrm{TeV}[11]$.

- The MSSM agrees with precision electroweak measurements. ${ }^{11}$

The fit to the electroweak precision data can be performed in the context of the MSSM and is shown in Fig. 5 for supersymmetric partner masses below $2 \mathrm{TeV}$. The MSSM with supersymmetric partner particles in the $1-2 \mathrm{TeV}$ region is actually a slightly better statistical fit to the data than the Standard Model. ${ }^{14}$

There are also many negative things about the supersymmetric model, the most obvious of which is: Where is it?

In the MSSM, the lightest Higgs boson mass has a theoretical upper bound,

$$
\begin{aligned}
M_{H}^{2}< & M_{Z}^{2} \cos ^{2} 2 \beta \\
& +\frac{3 G_{F} M_{t}^{4}}{\sqrt{2} \pi^{2} \sin ^{2} \beta} \log \left(\frac{\tilde{m}_{t 1} \tilde{m}_{t 2}}{M_{t}^{2}}\right),
\end{aligned}
$$

where $\tan \beta$ is the ratio of the neutral Higgs boson vacuum expectation values. Requiring that the Higgs boson mass satisfy the LEP direct search limit, $M_{H}>114 \mathrm{GeV}$, implies that the stop squarks must be relatively heavy, ${ }^{12}$

$$
\tilde{m}_{t 1} \tilde{m}_{t 2}>(950 \mathrm{GeV})^{2} .
$$

However, the supersymmetric partner particles in the MSSM are naturally on the order of the weak scale, so there is a tension between the desire for them to be light (to fill their required role in cancelling the quadratic contributions to the Higg boson mass as in Eq. 6 ) and the limit of Eq. 8. 


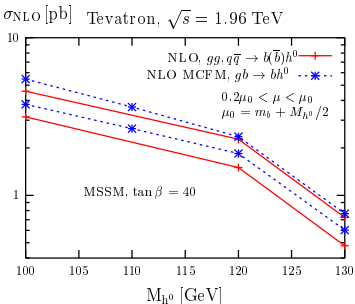

Figure 6. Total next-to-leading order cross section in the MSSM for $b H$ production at the Tevatron. The bands show the renormalization/factorization dependence. The solid (red) curves correspond to the fourflavor number scheme with no $b$ partons, and the dotted (blue) curves are the prediction from the fiveflavor number scheme with $b$ partons in the initial state[15].

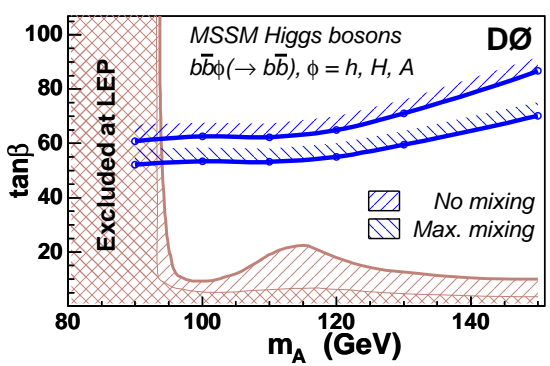

Figure 7. $95 \%$ c.l. upper limit from the D0 experiment at the Tevatron on $\tan \beta$ in the MSSM from $g g \rightarrow b \bar{b} \phi$, where $\phi$ is any of the three neutral Higgs bosons of the MSSM[17].

The couplings of the Higgs boson to the bottom quark are enhanced in the MSSM for large values of $\tan \beta$ and the dominant production mechanism becomes $g g \rightarrow b \bar{b} H$, where 0,1 , or $2 b$ quarks are tagged. ${ }^{15,16}$ Fig. 6 shows the total next-to-leading order cross section for $b H$ production at the Tevatron as a function of the mass of the lightest Higgs boson of the MSSM for $\tan \beta=40 .{ }^{15}$ D0 has a new limit on this process, which is shown in Fig.7. ${ }^{17}$

Many variants of the MSSM have been constructed. One of the simplest is the NMSSM (next-to-minimal- supersymmetric model) which is obtained by adding a Higgs singlet superfield $\hat{S}$ to the MSSM. ${ }^{18,19}$ The superpotential in the NMSSM is,

$$
W=W_{M S S M}+\lambda \hat{H}_{1} \hat{H}_{2} \hat{S}+\frac{\kappa}{3} \hat{S}^{3},
$$

where $\hat{H}_{1}$ and $\hat{H}_{2}$ are the Higgs doublet superfields of the MSSM, and $\hat{S}$ is the Higgs singlet superfield. When the scalar component of the singlet, $S$, gets a vacuum expectation value, the term $\lambda \hat{H}_{1} \hat{H}_{2}\langle S>$ in the superpotential naturally generates the $\mu \hat{H}_{1} \hat{H}_{2}$ term of the MSSM superpotential and it is straightforward to understand why $\mu \sim M_{Z}$. This is the major motivation for constructing the NMSSM.

In the NMSSM model, the bound on the lightest Higgs boson mass becomes,

$$
\begin{aligned}
M_{H}^{2}< & M_{Z}^{2} \cos ^{2} 2 \beta+v^{2} \lambda^{2} \sin ^{2} 2 \beta \\
& +1 \text {-loop corrections }
\end{aligned}
$$

and the lightest Higgs boson can be significantly heavier than in the MSSM. If we further assume that the couplings remain perturbative to the GUT scale, the theoretical upper bound on the lightest Higgs boson mass becomes $M_{H}<150 \mathrm{GeV} .^{20}$

The phenomenology in the NMSSM is significantly different than in the MSSM. There are three neutral Higgs bosons and two pseudoscalar Higgs bosons. A typical scenario for the masses is shown in Fig. 8. New decays such as the Higgs pseudoscalar into two scalar Higgs bosons are possible and changes the LHC Higgs search strategies. In addition, the lightest Higgs boson can have a large $\mathrm{CP}$-odd component and so can evade the LEP bound on $M_{H} \cdot{ }^{18,19}$

The minimal version of the MSSM conserves $\mathrm{CP}$, but $\mathrm{CP}$ violation in the Higgs sector can easily be accommodated in the MSSM. Non-zero phases in the scalar trilinear couplings can generate large CP violating effects from radiative corrections, especially those involving the third generation. If there is $\mathrm{CP}$ violation in the Higgs sector of the MSSM, then the three neutral Higgs mass 


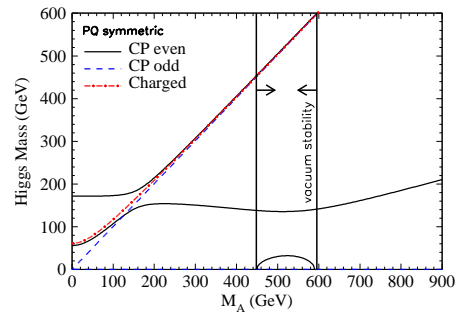

Figure 8. Typical mass scenario for the Higgs bosons in the NMSSM with $\tan \beta=3$. The region between the vertical lines (denoted by arrows) is the region allowed by vacuum stability[19].

eigenstates, $H_{1}, H_{2}$, and $H_{3}$, are mixtures of the CP- even and CP- odd Higgs states. ${ }^{21}$ The production and decay properties of the Higgs bosons can be very different from those of the Higgs bosons in the $\mathrm{CP}$ conserving version of the MSSM since the CP- odd components of the Higgs mass eigenstates do not couple to the $Z$ boson.

Experimental searches for the Higgs boson in a version of the MSSM with CP violation in the Higgs sector have been performed by the LEP collaborations ${ }^{22}$ using the benchmark parameters of the CPX model. ${ }^{21}$ For large values of $M_{H_{2}}, H_{1}$ is almost completely $\mathrm{CP}$ - even and the exclusion limit for the lightest Higgs boson mass is similar to the $\mathrm{CP}$ conserving limit. If $M_{H_{2}}>130 \mathrm{GeV}$, then $M_{H_{1}}>113 \mathrm{GeV}$. For lighter $M_{H_{2}}$, the $H_{1}$ has a large mixture of the CP- odd component and the result is that there are unexcluded regions in the $M_{H_{1}}-\tan \beta$ parameter space and the excluded region disappears completely for $4<\tan \beta<10$. At $95 \%$ c.l., $\tan \beta<3.5$ and $M_{H_{1}}<114 \mathrm{GeV}$ and also $\tan \beta>2.6$ are excluded in the CPX scenario. $^{a}$

It is interesting to compare the excluded regions in the $M_{H_{1}}-\tan \beta$ plane for the $\mathrm{CP}$ conserving and $\mathrm{CP}$ nonconserving versions of the MSSM, as shown in Figs. 9 and 10. We observe that the shape of the excluded region is significantly different in the two cases. As

${ }^{a}$ These limits assume $M_{t}=179.3 \mathrm{GeV}[22]$.

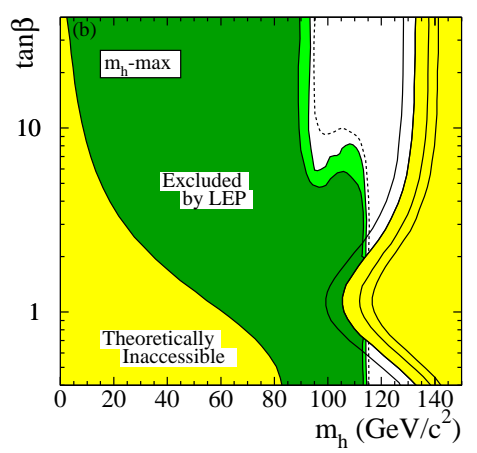

Figure 9. Excluded region in the $M_{H_{1}}$ - $\tan \beta$ plane in the CP conserving version of the MSSM. The light (dark) green is the $95 \%(97 \% \mathrm{cl})$ exclused region in the $M_{H}(\max )$ benchmark scenario. The solid lines from left to right vary the top quark mass: $M_{t}=$ $169.3,174.3,179.3$ and $183 \mathrm{GeV}[21]$.

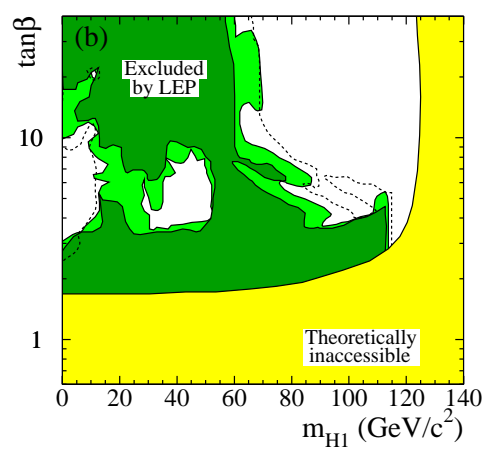

Figure 10. Excluded region in the $M_{H_{1}}-\tan \beta$ plane in the CPX CP violating version of the MSSM. The curves are as in Fig. 9[21].

noted in Ref.[22], the limit is extremely sensitive to small variations in the top quark mass.

\section{$5 \quad$ Little Higgs Models}

Little Higgs models ${ }^{7,8}$ are an attempt to address the hierarchy problem by cancelling the quadratic contributions to the Higgs boson mass in the Standard Model with the contributions resulting from the addition of new particles which are assumed to exist at a scale around $1-3 \mathrm{TeV}$. The cancellation of the quadratic contributions occurs between states with the same spin statistics. Thus 


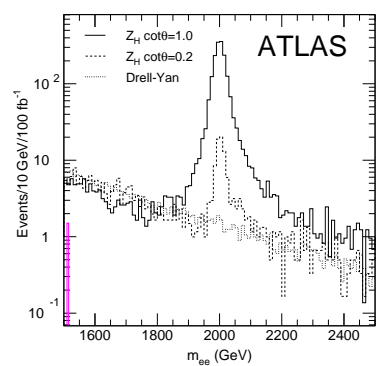

Figure 11. ATLAS simulation with $300 \mathrm{fb}^{-1}$ of data of the $e^{+} e^{-}$invariant mass distribution in a Little Higgs model resulting from the decay $Z_{H} \rightarrow Z H$ for $Z_{H}=2 \mathrm{TeV}$. The lower dotted histogram is the background[26].

contributions to Eq. 5 from the Standard Model $W, Z$, and photon are cancelled by the contributions from new heavy gauge bosons, $W_{H}, Z_{H}$ and $A_{H}$, with Standard Model quantum numbers, while Standard Model contributions from the top quark are cancelled by those from a heavy charge $2 / 3$ top-like quark, and those from the Higgs doublet by contributions from a scalar triplet. A clear prediction of the Little Higgs models is the existence of these new particles. Decays such as $Z_{H} \rightarrow Z H$ should be particularly distinctive ${ }^{8}$ as demonstrated in Fig.11. ${ }^{26}$

The basic idea of the Little Higgs models is that a continuous global symmetry is broken spontaneously and the Higgs boson is the Goldstone boson of the broken symmetry. There are many variants of this idea, with the simplest being a model with a global $\mathrm{SU}(5)$ symmetry broken to a global $\mathrm{SO}(5)$ symmetry by the vacuum expectation value of a non-linear sigma field $\Sigma=\exp (2 i \Pi / f)$. The Goldstone bosons contain both a Higgs doublet and a Higgs triplet and reside in the field $\Pi$. The parameter $f$ sets the scale of the symmetry breaking, which occurs at a scale $\Lambda \sim 4 \pi f \sim 10 \mathrm{TeV}$ where the theory becomes strongly interacting. The quadratic contributions to the Higgs boson mass of the Standard Model are cancelled by the new states at a scale $g f \sim 1-3 \mathrm{TeV}$. Further-

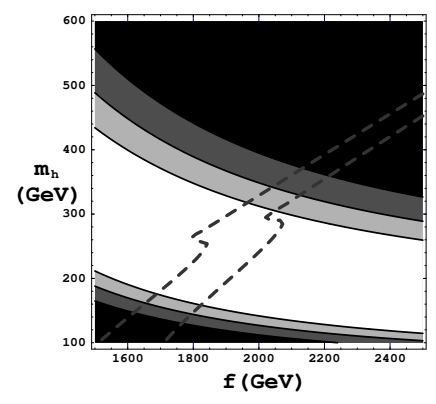

Figure 12. Excluded regions at 95\%, 99\% and $99.9 \%$ confidence level (from lightest to darkest) in the little Higgs model with T-Parity. In the band between the two dashed lines the lightest T-Parity odd particle is a consistent dark matter candidate and contributes to a relic density within $2 \sigma$ of the WMAP data[25].

more, the gauge symmetries are arranged in such a manner that the Higgs boson gets a mass only at two-loops, $M_{H} \sim g^{2} f /(4 \pi)$, and so the Higgs boson is naturally light, as required by the precision electroweak data.

The mixing of the Standard Model gauge bosons with the heavy gauge bosons of Little Higgs models typically gives strong constraints on the scale $f>1-4 T e V .{ }^{23}$ It is possible to evade many of these limits by introducing a symmetry ( $T$ parity) which requires that the new particles be produced in pairs. ${ }^{24,25}$ This allows the scale $f$ to be as low as $500 \mathrm{GeV}$. The lightest particle with $T$-odd parity is stable and is a viable dark matter candidate for $M_{H}$ between around 200 and $400 \mathrm{GeV}$ and the scale $f$ in the $1-2 \mathrm{TeV}$ region, as seen in Fig. 12.

Little Higgs models allow the lightest neutral Higgs boson to be quite heavy, as is demonstrated in Fig. $13 .^{27}$ The relaxation of the strong upper bound on the Higgs mass of the Standard Model is a generic feature of models with Higgs triplets.

\section{Higgsless Models}

Finally, we consider a class of models in which the Higgs boson is completely removed from the theory. These models face a number of 


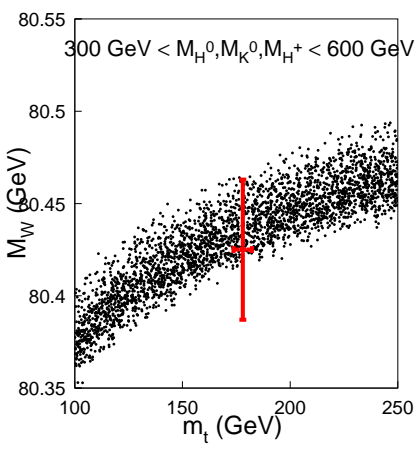

Figure 13. Predictions for the $W$ mass as a function of the top quark mass in a theory with a Higgs triplet. The masses of the three scalars in the theory, $H^{0}, K^{0}$, and $H^{ \pm}$, are varied between 300 and $600 \mathrm{GeV}$. The red point is the experimental data point with the $1 \sigma$ errors[27].

basic challenges:

- How to break the electroweak symmetry?

- How to restore unitarity without a Higgs boson?

- How to generate gauge boson and fermion masses?

- How to ensure

$$
\rho=\frac{M_{W}^{2}}{M_{Z}^{2} \cos ^{2} \theta_{W}}=1 ?
$$

Models with extra dimensions offer the possibility of removing the Higgs boson from the theory and generating the electroweak symmetry breaking from boundary conditions on the branes of the extra dimensions. ${ }^{6}$ Before even constructing such a Higgsless model, it is obvious that models of this class will have problems with the electroweak precision data. As can be seen from Fig.14, as the Higgs boson gets increasingly massive, the predictions of the Standard Model get further and further away from the data. A heavy Higgs boson gives too large a value of $S$ and too small a value of $T$. This figure gives a hint as to what the solution must eventually be: The Higgsless models must have a large

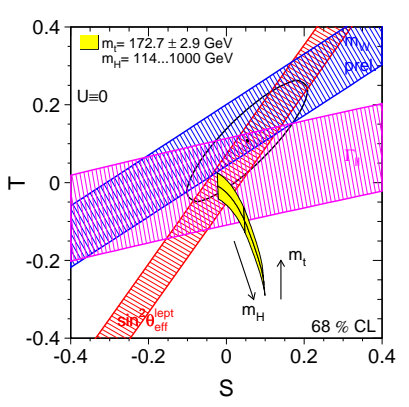

Figure 14. Limits on $S$ and $T$ from precision electroweak measurements, as of September, 2005. The Standard Model reference values (which give $S=$ $T=U=0)$ are $M_{t}=175 \mathrm{GeV}$ and $M_{H}=$ $150 \mathrm{GeV} \cdot[1]$

and positive contribution to $T$ and must not have any additional contributions to $S .{ }^{28}$

The Higgsless models all contain a tower of Kaluza Klein $(\mathrm{KK})$ particles, $V_{n}$, with the quantum numbers of the Standard Model gauge bosons. The lightest particles in the KK tower are the Standard Model $W, Z$, and $\gamma$. These Kaluza Klein particles contribute to the elastic scattering amplitudes for gauge bosons. In general, the elastic scattering amplitudes have the form, (where $E$ is the scattering energy):

$$
A=A_{4} \frac{E^{4}}{M_{W}^{4}}+A_{2} \frac{E^{2}}{M_{W}^{2}}+A_{0}+\ldots
$$

In the Standard Model, $A_{4}$ vanishes by gauge invariance and $A_{2}$ vanishes because of the cancellation between the gauge boson and Higgs boson contributions. In the Higgsless models, the contributions to $A_{4}$ and $A_{2}$ cancel if,

$$
\begin{aligned}
g_{n n n n}^{2} & =\Sigma_{k} g_{n n k}^{2} \\
4 g_{n n n n}^{2} & =3 \Sigma_{k} g_{n n k}^{2} \frac{M_{k}^{2}}{M_{n}^{2}},
\end{aligned}
$$

where $g_{n n k}$ is the cubic coupling between $V_{n}$, $V_{n}$, and $V_{k}, g_{n n n n}$ is the quartic self coupling of $V_{n}$, and $M_{k}$ is the mass of the $k^{t h} \mathrm{KK}$ particle. 


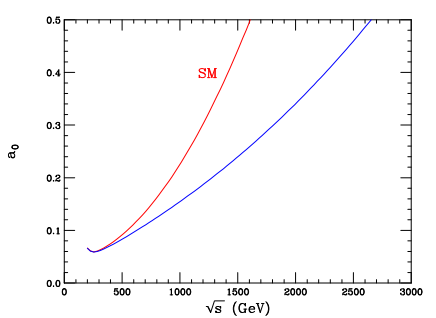

Figure 15. $J=0$ partial wave for elastic gauge boson scattering in the Standard Model with the Higgs boson removed (red) and with the inclusion of a single Kaluza Klein excitation with $M=500 \mathrm{GeV}$ (blue) in a deconstructed Higgsless model[30].

The amazing fact is that the 5dimensional Higgsless models satisfy these sum rules exactly due to 5 -dimensional gauge invariance. Similarly, 4-dimensional deconstructed versions of the Higgsless models ${ }^{33}$ satisfy these sum rules to an accuracy of a few percent. The Kaluza Klein particles play the same role as the Higgs boson does in the Standard Model and unitarize the scattering amplitudes. Of course, the lightest Kaluza Klein mode needs to be light enough for the cancellation to occur before the amplitude is already large, which restricts the masses of the Kaluza Klein particles to be less than $1-2 \mathrm{TeV} \cdot{ }^{29,30}$

Fig. 15 shows the growth of the $J=0$ partial wave in the Standard Model with the Higgs boson removed and in a Higgsless model with a single Kaluza Klein particle with mass $M=500 \mathrm{GeV}$ included. The inclusion of the Kaluza Klein contributions pushes the scale of unitarity violation from $E \sim 1.6 \mathrm{GeV}$ in the Standard Model with no Higgs boson to around $E \sim 2.6 \mathrm{TeV}$ in the Higgsless models.

The Kaluza Klein particles contribute to the electroweak precision measurements. In general, the corrections are too large for KK particles with masses on the $\mathrm{TeV}$ scale. ${ }^{31}$ Considerable progress in addressing this problem has been made in the last year with the realization that the contributions of the Kaluza Klein particles to the precision
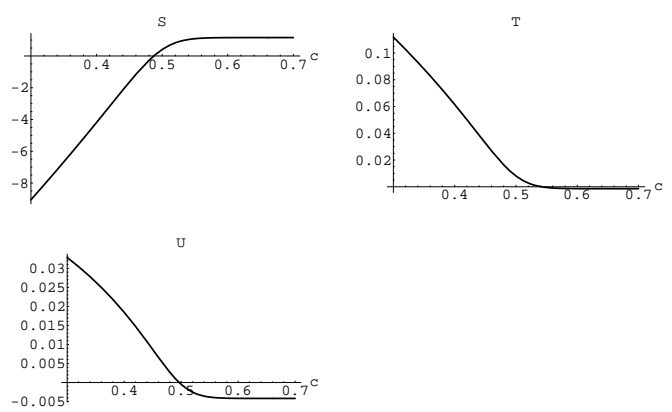

Figure 16. Oblique parameters, $S, T$, and $U$ in a Higgsless model as a function of the fermion localization parameter, $c$. If the fermions are localized on the $\mathrm{TeV}$ brane, $c<<\frac{1}{2}$, while fermions localized on the Planck brane have $c>>\frac{1}{2}$. A flat fermion wavefunction corresponds to $c=\frac{1}{2}[32]$.

electroweak observables depend on where the fermions are located in the extra dimensions. In the Randall-Sundrum model, $S$ is positive if the fermions are located on the Planck brane and negative if they are located on the $T e V$ brane. The trick is to find an intermediate point where there is a weak coupling between the KK modes and the fermions. ${ }^{31,32}$ It appears to be possible to construct models which are consistent with the electroweak precision measurements by having the fermion wavefunction be located between the branes. ${ }^{32}$

Fig. 16 shows the oblique parameters as a function of the variable $c$, which characterizes the location of the fermion wavefunction. If the fermions are localized on the $\mathrm{TeV}$ brane, $c<<\frac{1}{2}$, while fermions localized on the Planck brane have $c>>\frac{1}{2}$. A flat fermion wavefunction corresponds to $c=\frac{1}{2}$. For $c \sim 1 / 2$ it is possible to satisfy the bounds from precision electroweak data. Fermions with a flat wavefunction are weakly coupled to the Kaluza Klein particles and so such Kaluza Klein particles would have escaped the direct searches for heavy resonances at the Tevatron.

The next challenge for Higgsless models is to generate the large mass splitting be- 
For Publisher's use

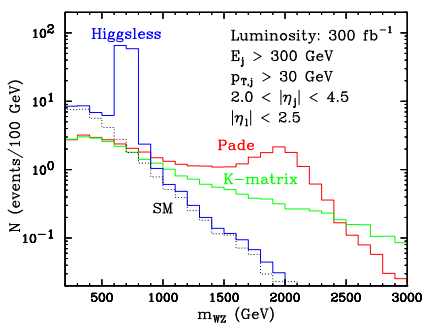

Figure 17. The number of events per $100 \mathrm{GeV}$ bin in the 2 -jet plus 3 lepton plus $\nu$ channel at the LHC, coming from the subprocess $W Z \rightarrow W Z$ in a Higgsless model[34].

tween the top and the bottom quarks. ${ }^{35}$

Weakly coupled Kaluza Klein particles are a generic feature of Higgsless models and can be searched for in a model independent fashion. These KK particles appear as massive $W-, Z$-, and $\gamma$ - like resonances in vector boson fusion and they will appear as narrow resonances in the $W Z$ channel as shown in Fig. $17 .^{34}$ The lightest KK resonance should be clearly observable above the background.

\section{Conclusions}

The mechanism of electroweak symmetry breaking could be far more complicated than a simple Higgs boson. Almost all models, however, have distinctive signatures which should be observed at the LHC. Soon, with data from the LHC, we should have some indication what mechanism nature has chosen! A complete understanding of the unknown physics awaiting us at the $\mathrm{TeV}$ scale will probably require a future linear collider. ${ }^{36}$

\section{Acknowledgments}

This research supported by Contract No. DE-AC02-76CH1-886 with the U.S. Department of Energy. I thank my collaborators, M.C. Chen, C. Jackson, T. Krupovnickas, L. Reina, and D. Wackeroth for countless discussions.

\section{References}

1. LEP Electroweak Working Group, http://lepewwg.web.cern.ch /LEPEWWG/.

2. Preliminary result posted at http://wwwcdf.fnal.gov/physics/exotic.html.

3. B. Lee, C. Quigg, and H. Thacker, Phys. Rev. D 16, 1519 (1977); D. Dicus and V. Mathur, Phys. Rev. D 7, 3111 (1973)

4. G. Giudice, Int. J. Mod. Phys. A 19, 835 (2004), hep-ph/0311344; R. Barbieri, A. Pomerol, and A. Strumia, Nucl. Phys. B 703, 127 (2004), hep$\mathrm{ph} / 0405040$.

5. Z. Han and W. Skiba, Phys. Rev. D 71, 075009, hep-ph/0412166.

6. C. Csaki, C. Grojean, H. Murayama, L. Pilo, and J. Terning, Phys. Rev. D 69, 055006 (2004), hep-ph/0305237; C. Csaki, C. Grojean, L. Pilo, and J. Terning, Phys. Rev. Lett. 92, 101802 (2004), hep-ph/0308038; R. Chivukula, E. Simmons, H.-J. He, M. Kurachi, and M. Tanabashi, Phys. Rev D 70, 075008 (2004), hep-ph/0406077. For a review and references to the original literature see G. Cacciapaglia, C. Csaki, C. Grojean,J. Terning, eConf C040802:FRT004, 2004, C. Csaki, hep$\mathrm{ph} / 0412339$.

7. N. Arkani-Hamed, A.Cohen, E. Katz, and A. Nelson, JHEP 0207, 034 (2002), hep-ph/0206021; N. ArkaniHamed, A.Cohen, E. Katz, A. Nelson, T. Gregoire, and J. Wacker, JHEP 0208, 021 (2002), hep-ph/0206020; D. Kaplan and M. Schmaltz, JHEP 0310, 039 (2003), hep-ph/0302049; W. Skiba and J. Terning, Phys. Rev. D 68, 075001 (2003), hep-ph/0305302.

8. For a review and references to the original literature, see T. Han, H. Logan, and L.-T. Wang, hep-ph/0506313.

9. R. Harnik, G. Kribs, D. Larson, and 
H. Murayama, Phys. Rev. D 70, 015002 (2004), hep-ph/0311349.

10. C. Hill and E. Simmons, Phys. Rept 381, 235 (2003), hep-ph/0203079.

11. S. Heinemeyer, W. Hollik, and G. Weiglein, hep-ph/0412214.

12. R. Dermisek and J. Gunion, Phys. Rev. Lett.95, 041801 (2005), hep-ph/0502105.

13. M.Drees, hep-ph/9611409 and S. Dawson, TASI97, hep-ph/9712464, and references therein.

14. W. deBoer and C. Sander, Phys. Lett. B 585, 276 (2004), hep-ph/0307049.

15. S. Dawson, C.Jackson, L. Reina, and D. Wackeroth, hep-ph/0508293.

16. S. Dawson, C.Jackson, L. Reina, and D. Wackeroth, Phys. Rev. Lett. 94, 031802 (2005), hep-ph/0408077; J. Campbell et. al., hep-ph/0405302; S. Dittmaier, M. Kramer, and M. Spira, Phys. Rev. D 70, 074010 (2004); F. Maltoni, Z. Sullivan, and S. Willenbrock, Phys. Rev. D 67, 093005 (2003), hep-ph/0301033.

17. D0 Collaboration, Phys. Rev. Lett. 95, 151801 (2005).

18. U. Ellwanger, J. Gunion, and C. Hugonie,JHEP 0507, 041 (2005), hepph/0503203; D. Miller, R. Nevzorov, and P. Zerwas, Nucl. Phys. B 681, 3 (2003), hep-ph/0304049.

19. U. Ellwanger, J. Gunion, C. Hugonie, and S. Moretti, hep-ph/0401228.

20. G. Kane, C. Kolda, and J. Wells, Phys. Rev. Lett. 70, 2686 (1993), hepph/9210242; M. Quiros and J. Espinosa, hep-ph/9809269.

21. M. Carena, J. Ellis, A. Pilaftsis, C. Wagner, Phys. Lett. B 495, 155 (2000); Nucl. Phys. B 586, 92 (2000).

22. ALEPH, DELPHI, L3, and OPAL Collaborations, Search for Neutral Higgs Bosons at LEP, LHWG-Note-2005-01.

23. J.

Hewett, F. Petriello, and T. Rizzo, JHEP0310, 062 (2003), hep-ph/0211218; C. Csaki,
J. Hubisz, P. Meade, and J. Terning, Phys. Rev. D 68, 035009 (2003), hep-ph/0303236; R. Casalbuoni, A. Deandrea, and M. Oertel, JHEP 0402, 032 (2004), hep-ph/0311038; W. Kilian and J. Reuter, Phys. Rev. D 70, 015004(2004), hep-ph/0311095; M. Chen and S. Dawson, Phys. Rev. D 70,015003 (2004), hep-ph/0311032; T. Gregoire, D. Smith and G. Wacker, Phys. Rev. D 69,115008 (2004), hepph/0305275.

24. H. Cheng and I. Low, JHEP 0408, 061 (2004), hep-ph/0405243; I. Low, JHEP 0410, 067 (2004), hep-ph/0409025; J. Hubisz and P. Meade, Phys. Rev. D 71, 035016 (2005), hep-ph/0411264.

25. J. Hubisz, P. Meade, A. Noble, and M. Perelstein, hep-ph/0506042.

26. G. Azuelos et.al., Eur. Phys. Jour. C 39S2, 13 (2005), hep-ph/0402037.

27. T. Blank and W. Hollik, Nucl. Phys. B 514, 113 (1998), hepph/9703392; M. Chen, S. Dawson, and T. Krupovnickas, hep-ph/0504286.

28. R. Chivukula, C. Hoelbling, and N. Evans, Phys. Rev. Lett 85, 511 (2000), hep-ph/0002022; M. Peskin and J. Wells, Phys. Rev. D 64, 093003 (2001), hep-ph/0101342.

29. H. Davoudiasl, J. Hewett, B. Lillie, and T. Rizzo, JHEP 0405, 015 (2004), hepph/0403300;Phys. Rev. D 70, 015006 (2004); M. Papucci, hep-ph/0408058; R. Casalbuoni, S. DeCurtis, and D. Dominici, Phys. Rev. D 70, 055010 (2004), hep-ph/0405188.

30. R. Foadi, S. Gopalakrishna, and C. Schmidt, JHEP 403, 042 (2004), hep-ph/0312324.

31. K. Agashe, A. Delgado, M. May, R. Sundrum, JHEP 0308, 050 (2003), hepph/0308036; J. Hewett, B. Lillie, and T. Rizzo, JHEP 0410, 014 (2004), hepph/0407059; R. Foadi, S. Gopalakrishna, and C. Schmidt, Phys. Lett. B 606, 157 
(2005), hep-ph/0409266.

32. C. Cacciapaglia, C. Csaki, C. Grojean, J. Terning, Phys. Rev. D 71, 035015 (2005), hep-ph/0409126.

33. R. Chivukula, H.-J. He, M. Kurachi, E. Simmons, and M. Tanabachi, Phys. Rev. D 72, 015008 (2005), hepph/0504114; R. Chivukula, H.-J. He, M. Kurachi, E. Simmons, and M. Tanabachi, Phys. Rev. D 70, 075008 (2004), hep-ph/0406077; R. Chivukula, H.-J. He, M. Kurachi, E. Simmons, and M. Tanabachi, Phys. Lett. B 603, 210 (2004), hep-ph/0408262.

34. A. Birkedal, K. Matchev, and M. Perelstein, Phys Rev. Lett. 94, 191803 (2005).

35. G. Cacciapaglia, C. Csaki, C. Grojean, M. Reece, and J. Terning, hepph/0505001; R. Foadi and C. Schmidt, hep-ph/0509071; H. Davoudiasl, B. Lillie, and T. Rizzo, hep-ph/0508279; R. Chivukula, H.-J. He, M. Kurachi, E. Simmons, and M. Tanabachi, hep$\mathrm{ph} / 0508147$.

36. S. Dawson and M. Oreglia, Ann. Rev. Nucl. Part. Sci. 54, 269 (2004), hep$\mathrm{ph} / 0403015$.

\section{DISCUSSION}

Daniel Kaplan (Illinois Institute of Technology):

How does the new state possibly seen in the HyperCP experiment at Fermilab fit into SUSY models? It has a mass of $214.3 \mathrm{MeV}$ and decays into $\mu^{+} \mu^{-}$.

Sally Dawson: This state is very difficult to understand in terms of SUSY models.

Anna Lipniacka (University of Bergen):

Is gauge coupling unification natural in Large Extra Dimension models?

Sally Dawson: No. These theories typically violate unitarity and become strongly interacting at a scale between 1 and $10 \mathrm{TeV}$.

\section{Ignatios Antoniadis (CERN):}

What is the prize to pay in models that solve the little hierarchy problem, such as the little Higgs models, in particular on the number of parameters and the unification of gauge couplings?

Sally Dawson: Obviously, there is a large increase in the number of parameters and gauge unification is forfeited.

Luca Silvestrini (Munich and Rome): Maybe one should comment about the statement that you made that new physics has to have a scale $\Lambda$ greater than $5 \mathrm{TeV}$. Of course this is a conventional scale that is only valid if the coupling in front of the operator is one, which is generally not true in any weakly interacting theory and generally not true if new physics enters through loops. So I do not want that anybody in the audience really believes that new physics must be at a scale larger than $5 \mathrm{TeV}$. It can easily be around the electroweak scale as we know very well.

Sally Dawson: Absolutely true. The limits depend on the couplings to the operators, which in turn depend on the model. 